\title{
Modeling And Performance Analysis Of Marine DTN Networks With Nodes- Cluster In An Ad Hoc Sub-Net
}

\author{
Yao Shi, Hui Li, Wen-Cai Du \\ College of Information Science and Technology, Hainan University, \\ Haikou P. R. China \\ E-mail: 83035648@qq.com, lihui@hainu.edu.cn,wencai@hainu.edu.cn
}

\author{
Ji-Xin Ma \\ Department of Computing and Information Systems \\ University of Greenwich, UK \\ E-mail: j.ma@gre.ac.uk
}

\author{
Fa-Bin Li \\ 63812 Troop of PLA \\ Wenchang, P. R. China \\ E-mail: fabinl@163.com
}

\begin{abstract}
The marine communication environment is complex and changeable, and the sea wireless infrastructure is few. The delay tolerant network is proposed and applied in ocean environment. We use the ships to store, carry and forward the messages, so as to solve the problem that the communication links are broken frequently and the message cannot be transmitted due to the movement of the nodes. Under the environment of MATLAB, the random motion trajectory of a fishing vessel in the South China Sea is modeled and simulated. A mobile Ad hoc network is formed between ship and ship, and the base station of the land mobile communication network is connected with the marine radio transmitting point through optical fiber. Data is generated according to Poisson distribution in a heterogeneous network at sea and the data transmission uses the flooding mechanism. Taking into account the limited capacity of the wireless transmission point of the sea, we introduced a forwarding-time-limited mechanism. The performance of it is analyzed in the delayed network at sea, and the effects of the survival time, the number of fishing vessels and the coverage of the wireless network on data delivery are compared. The simulation results show that increasing the survival time of the data and the coverage of the wireless network can improve the data delivery ratio, reduce the network transmission delay, and improve the performance of the maritime wireless communication network.
\end{abstract}

Keywords-delay tolerant network; Poisson distribution; mobile Ad hoc network; survival time; packet delivery ratio; transmission delay

\section{INTRODUCTION}

Due to the complexity of the ocean environment, the difficulty in the deployment of fixed wireless infrastructure and electromagnetic interference at sea, many advanced terrestrial wireless communication technologies are difficult to be applied to the marine environment, resulting in the development of marine communication is relatively backward. Inshore fishing vessels can make use of the cellular mobile communication network to receive data; on the contrary, offshore fishing can only rely on the maritime satellite to obtain information. However, due to the high cost of satellite communications, ship-based satellite stations are rarely used for inter-ship communication. The mobility of fishing vessels at sea causes the communication link to break frequently and the message cannot be transmitted timely. In $[1,2]$, it is proposed that the cellular mobile communication network, the maritime ad hoc network and the satellite mobile communication network can be integrated into a maritime heterogeneous network by the corresponding gateway, which provides a wireless communication support for the ship. Mobile Ad Hoc Network (MANET) is a multihop network which is not set up by any preset infrastructure. It has the advantages of rapid networking and flexible structure. In [3-5], MANET is applied to marine communication, which provides a good platform for shipboard information transmission.

Delay Tolerant Network (DTN) is a class of networks which the communication links are interrupted frequently and end-to-end data transmission is intermittent. It is mainly due to the movement of nodes, sparse distribution, energy constraints and other reasons. DTN is a message-oriented overlay network, which adds a Bundle layer between the application layer and the transport layer of the traditional OSI (Open system interconnection) model ${ }^{[6]}$. Generally, the Bundle layer aggregates the data from the application layer, and gateway node uses the "storage - carry - forward" mode to implement the cross-network transmission of messages. Compared with the traditional network, it has the characteristics of dynamic topology change, large propagation delay, high packet loss ratio and low signal-tonoise ratio ${ }^{[7,8]}$.

At present, many research works of DTN mainly focus on routing protocols. Due to the uncertainty of transmission opportunities, the routing algorithm needs to select some nodes as relay which carry and forward data for other nodes $[9,10]$. A flood-based Epidemic routing algorithm was proposed in $[11,12]$. After the node receives the message, it saves the data and transmits it to the neighbor node. The neighbor node repeats this step until each node receives the message. In order to reduce the network overhead, a hoplimited Epidemic routing algorithm is proposed in [13]. This algorithm can achieve better network performance by controlling the number of hops in the message. The 
experimental results show that the message hop count has certain influence on routing performance. The robustness of the network is better when the number of nodes is larger. [14, 15] proposed a new routing scheme called Spray and Wait routing. In the Spray phase, the source node generates $\mathrm{N}$ message replicas and sends them to $\mathrm{N}$ neighbor nodes, after receiving the message, the node enters the Wait state; in the Wait phase, the node no longer replicates the message, but looks for the destination node to deliver the message. The message delivery ratio of the routing algorithm depends on the number of copies of the message in the Spray phase and the probability that the nodes meet. In some cases, the node cache is occupied by invalid messages for a long time. In [16], the mobile connection routing algorithm is combined with the reciprocal counter and the cache release strategy to accelerate the cache release. This algorithm can improve the network transmission efficiency under the premise of ensuring reliable data transmission.

Maritime wireless communication environment and DTN network has many similarities. In this paper, DTN is applied to maritime communications, and a multi-network heterogeneous maritime wireless communication scheme is designed to solve the network disruption caused by node movement or sea environment change. To store, carry and forward information by means of fishing vessel. Taking into account the number and the storage capacity of sea surface wireless transmitting points, an Epidemic routing algorithm with anchor node forwarding time restriction is proposed to reduce the number of retransmissions of messages at sealevel wireless transmitting points and lighten the network burden. Then, the influence of the survival time, the number of nodes and the coverage of the wireless network on the communication performance in the established network model is compared by a large number of simulation experiments.

\section{NETWORK MODEL AND PROBLEM DESCRIPTION}

Wherever Times is specified, Times Roman or Times New Roman may be used. If neither is available on your word processor, please use the font closest in appearance to Times. Avoid using bit-mapped fonts if possible. True-Type 1 or Open Type fonts are preferred. Please embed symbol fonts, as well, for math, etc.

\section{A. Network Model}

Combined with the actual situation of a sea area of the South China Sea, a heterogeneous network with the fusion of multiple maritime wireless networks is constructed, which is shown in Figure 1. The base station of the mobile communication network is deployed in the offshore land; inbound and outbound vessels rely on cellular networks for communication. The wireless transmission towers (anchor nodes) at sea are sparsely distributed, and there is no overlap of network coverage. The base station and the anchor node are connected by optical fiber in order to share the data information. The command room is not scheduled to send weather or other information to the sea vessels, the generation of these messages follows the Poisson distribution
[17]. The probability of the number of message generation in unit time satisfies (1)

$$
P(X=k)=\frac{(\lambda)^{k} e^{-\lambda}}{k !}
$$

where $\lambda$ is the frequency of the message and $k$ is the number of times the message is generated. There is a large area of network coverage loophole in the sea, and the vessel travels between the network coverage area and coverage loopholes for offshore operations. Anchor node forwarding mechanism with Time To Live (TTL) limited is used in the network model, in order to control the transmission times of messages at anchor nodes. A mobile ad hoc network is formed between the ships, and the information is transmitted in a multi-hop manner.

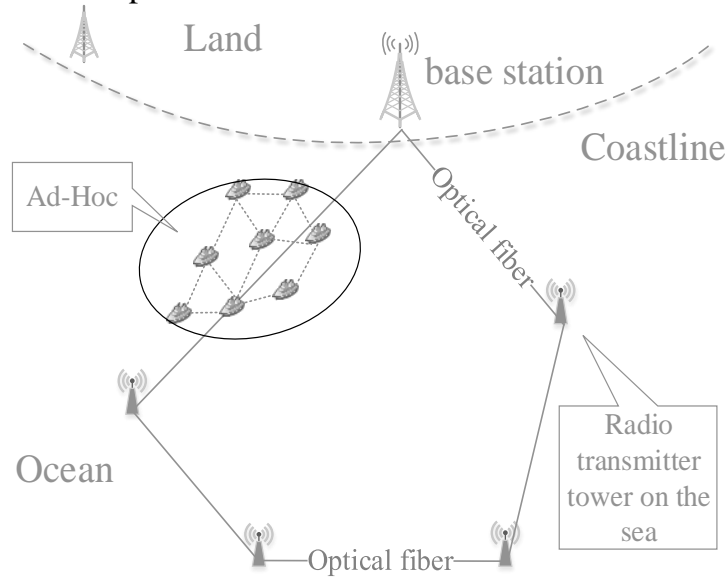

Figure 1. Heterogeneous Networks on the Sea.

\section{B. Process Description}

This section describes several cases of information delivery while ships are moving on the sea. In order to facilitate subsequent statements, the meaning of the relevant parameters involved in the paper is given first, as shown in Table I.

TABLE I. TABLE THE EXPLANATION OF RELEVANT SYMBOLS

\begin{tabular}{|c|c|}
\hline Parameter Settings & Parameter Meaning \\
\hline$S$ & Sea area \\
\hline$R$ & Communication range of anchor nodes \\
\hline$r$ & Communication range of vessel \\
\hline Nodes & Number of vessels \\
\hline$V_{\max }$ & Maximum movement speed of vessel \\
\hline$I$ & Number of messages generated \\
\hline$M$ & Number of messages delivered successfully \\
\hline$T_{-}$produce $(i)$ & Generation time of the message $i$ \\
\hline$T_{-}$spread $(i)$ & Spread time of the message $i$ \\
\hline$t t l$ & Time to live \\
\hline$Q$ & Delivery ratio \\
\hline$T$ & Average delay \\
\hline
\end{tabular}


Nodes ships sailing around the sea, whose area is $S$, moves to the target area? At different moment, each fishing vessel's course is random and the velocity is a random value between $\left[0, V_{m a x}\right]$. The ship remains relatively independent, which complies with the characteristics of the DTN network nodes. Information transmission includes the following three situations:

- Low latency transmission. Message is generated at T_produce $(i)$ and vessels according to their location to determine the nearest anchor node. In a life cycle $t t l$, if the number of vessels entering the network coverage area is large and the distribution of the ship is relatively dense (the spacing of them is not greater than $r$ ), it is conducive to message delivery. For this message, the waiting time delay is small.

- High delay transmission. If the number of vessels entering the network coverage area is less and the distance between them is more than $r$ in a life cycle, messages are queued and cannot spread to the adjacent vessels. The boats which received the message will send data until the distance is no more than $r$.

- Message loss. If no vessel receives a message within a life cycle, anchor node will discard the data to reduce power consumption. There is no copy of the message in the network, which causes the packet loss.

\section{Performance Evaluation}

Network performance is reflected by the ability of the network to transmit data ${ }^{[18-20]}$. In the maritime delay tolerant network, the dynamic changes of network topology caused by the random movement of fishing vessels have a great influence on the performance of the network. At the same time, the wireless communication range $R$ of the anchor node is limited due to the electromagnetic interference at sea. During the test time, vessels that appear in the blank area of the network may not be able to receive data. The amount of data received by the vessel is less than the total amount of data sent by the network. The network delivery ratio $Q$ satisfies (2)

$$
Q=\frac{M}{I}
$$

The network delay includes transmission delay, propagation delay, processing delay and queuing delay. For the above network model, the delay is mainly caused by the packet queuing. Therefore, the average delay $T$ of the maritime delay network satisfies (3)

$$
T=\frac{\sum_{i} T_{-} \operatorname{spread}(i)}{M}
$$

\section{RELATED ALGORITHMS}

Shortest path flooding algorithm with anchor node forwarding-time limited reduces the marine DTN network overhead and saves node energy. First, the data is forwarded to the fishing vessel which is covered by the anchor node; then the data is forwarded to all fishing vessels through the MANET which is established between boats, the pseudo code is shown in Table II.

\section{TABLE II. TABLE SHORTEST-PATH FLOODING ALGROITHM WITH} ANCHOR NODE FORWARDING-TIME LIMITE

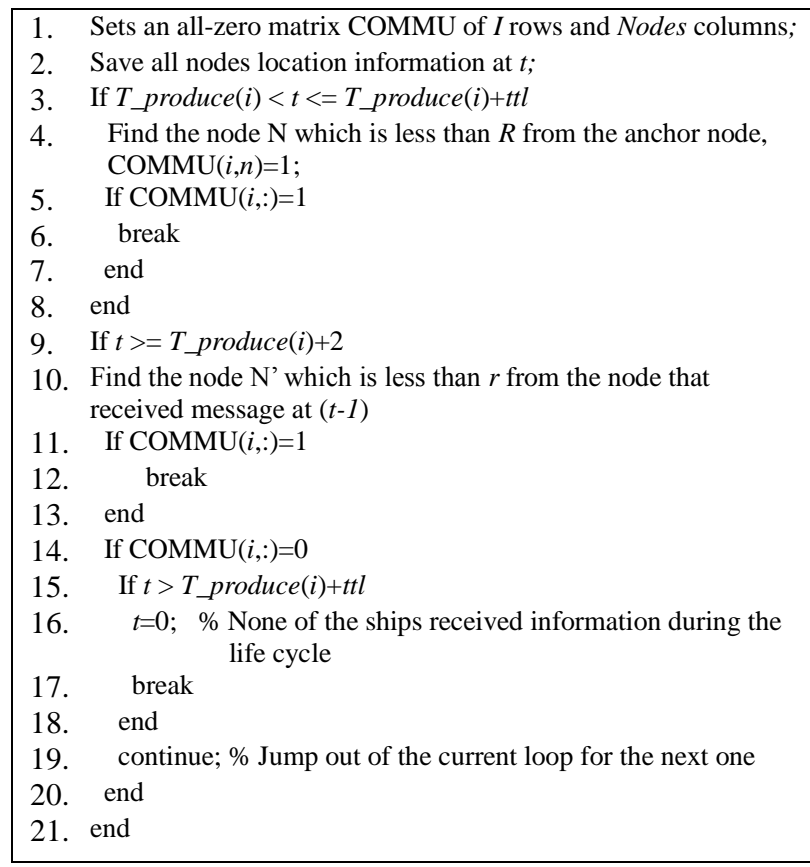

\section{NUMERICAL RESULTS ANALYSIS}

The proposed network model is modeled by MATLAB. Figure 2 shows the distribution of anchor nodes and the coverage of network, the specific parameters set as shown in Table III. On the basis of this, the influence of the number of fishing vessels, the communication distance of anchor nodes and the survival time of the data on the network are compared.

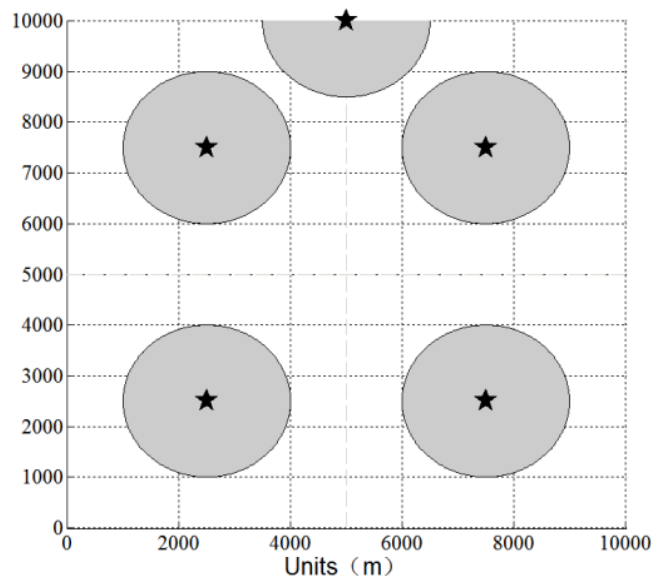

Figure 2. Distribution of Offshore Nodes. 
TABLE III. TABLE SIMULATION PARAMETER SETTING

\begin{tabular}{|c|c|}
\hline Parameter-setting & Parameter value \\
\hline$\lambda$ of Poisson distribution & 0.05 \\
\hline Simulation time $T(\mathrm{~s})$ & $>12000$ \\
\hline$S\left(\mathrm{~km}^{2}\right)$ & $10 \times 10$ \\
\hline Nodes & 10,20 \\
\hline$V_{\max }(\mathrm{m} / \mathrm{s})$ & 5 \\
\hline$t t l(\mathrm{~s})$ & $30,60,90,120,150,180$ \\
\hline$r(\mathrm{~m})$ & 100 \\
\hline$R(\mathrm{~m})$ & $1100,1300,1500$ \\
\hline
\end{tabular}

\section{A. The Influence of Node Coverage on Network}

The communication distance of node determines the size of the network coverage area. The generation time of the data packet is calculated by the formula (1). When $t t l=30 \mathrm{~s}$, according to the formula (2) and formula (3), the network delivery ratio and the average time delay are calculated. As can be seen in Figure 3, when the anchor node communication radius is equal to 1100 meters, 10 fishing vessels are corresponding to the network delivery ratio of $47.93 \%$, while 20 boats are corresponding to the network delivery ratio of $48.69 \%$. When the communication radius increases to 1500 meters, 10 fishing vessels are corresponding to the network delivery ratio as high as $59.40 \%, 20$ fishing vessels are corresponding to the delivery ratio of $60.72 \%$. In Figure 4, when the anchor node communication radius is 1100 meters, 10 fishing vessels are corresponding to the average network delay is $133.34 \mathrm{~s}, 20$ fishing vessels are corresponding to the average delay is $100.58 \mathrm{~s}$. As the radius increases, the network delay decreases to $55.47 \mathrm{~s}$ and $50.17 \mathrm{~s}$ respectively.

It can be seen that when the communication radius of nodes increases, the network delivery ratio increases, on the contrary, the average delay of the network decreases. For the MANET which is composed of vessels, increasing the number of nodes can effectively reduce the average time delay of the network, but it has little effect on the delivery ratio. The anchor node coverage is limited, and the possibility of fishing boats out of the cover hole is not affected by the number of nodes. The possibility is related to the TTL and its own movement speed. As the number of vessels increases, the node density becomes larger in MANET, and the distance between ship and ship decreases, which improves the efficiency of data forwarding and decreases the average delay. In the case of a fixed survival time, the performance of the network can be improved effectively by extending the communication range of the anchor node. It can be achieved by increasing the transmission power of the high-frequency carrier.

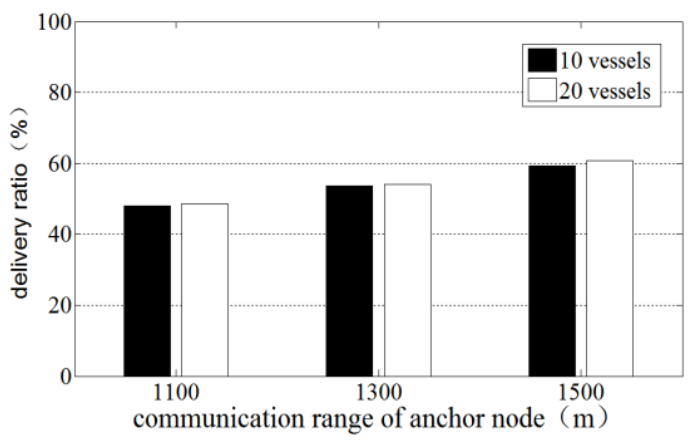

Figure 3. Effect of Anchor Node Radius on Delivery Ratio.

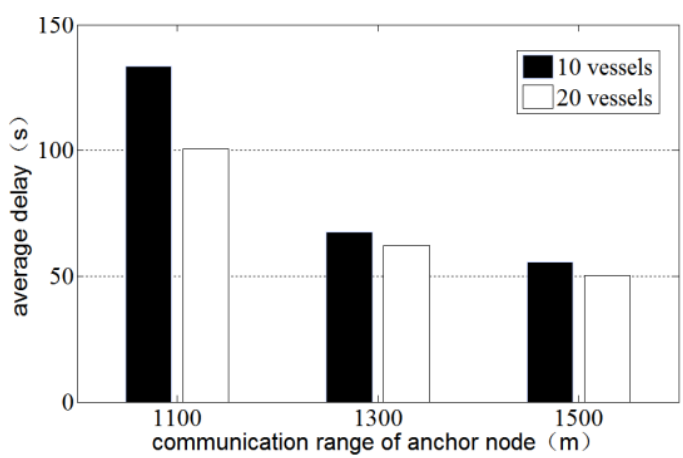

Figure 4. Effect of Anchor Node Radius on Average Delay.

\section{B. The Influence of TTL on Network}

The data survival time limits the forwarding time of anchor nodes. When the communication radius $(R)$ is 1500 meters and the number of mobile nodes (Nodes) is 20, the effect of different survival time on network performance is compared. As shown in Figure 5, when ttl is 30s, the network delivery ratio is as low as $60.72 \%$. The network delivery ratio shows an increasing trend when the survival time is increased by $30 \mathrm{~s}$, and the highest delivery ratio is $68.69 \%$ when $\mathrm{ttl}$ is 180 s. Prolonging the survival time will increase the probability of fishing vessels entering the network coverage area. The fishing vessel can carry and forward the message after receiving it in the process of moving, and the network delivery ratio can be promoted through this method. The network delay depends on the density of the fleet after the information is received by the fishing vessel. Dense distribution of fishing vessels is in favor of information transmission; on the contrary, sparse distribution will cause the data queuing, so that the survival time change has little effect on the network delay. As shown in Figure 6, the network latency fluctuates between 39.60s and $59.09 \mathrm{~s}$ under this condition, there is no obvious regularity in the graph, and the affected by the survival time is little. 


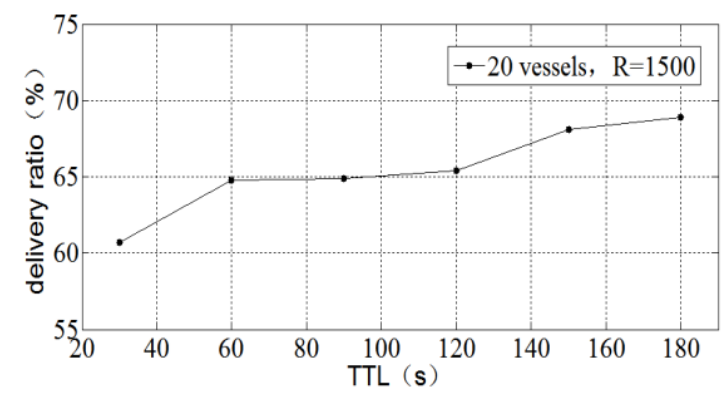

Figure 5. Effect of Anchor Node Radius on Delivery Ratio.

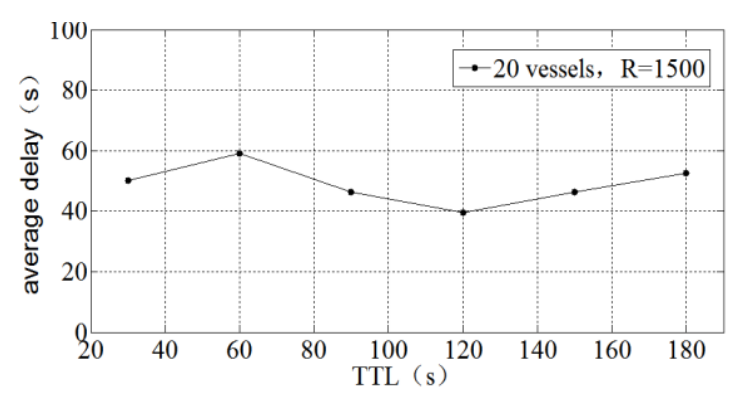

Figure 6. Effect of TTL on Average Delay.

\section{CONCLUSION}

Compared with the land mobile communication, the marine communication environment is relatively bad, and the infrastructure such as the base station is more difficult to deploy. In some hot spots, the electromagnetic environment is complex, thus there is a large area network coverage holes on the sea, which leads to the long time break of the communication link. Aiming at this problem, this paper proposes the application of delay tolerance network to marine environment and a routing algorithm with anchor node forwarding-time limited.

A large quantity of simulation data show that increasing the anchor node communication distance and prolonging the survival time of the data can improve the network delivery ratio and reduce the average delay. In order to communicate conveniently and effectively between the ships, and also to reduce the communication cost, it is possible to weigh the data volume, node storage capacity and node energy consumption on the basis of the shortest path flooding algorithm with anchor node forwarding-time limited. According to the needs of actual application and setting the appropriate data survival time, both to enhance network performance and reduce node energy consumption. DTN has the practical value in the marine communication in the future. It can alleviate the problem of resource information sharing and improve the ability to quickly respond to natural disasters and emergencies.

In the following study, the unmanned ship can be considered as a mobile anchor node, which can reduce the blind area of network coverage, to provide secondary relay for data forwarding and improve the network delivery ratio. At the same time, the research of anycast routing algorithm is also carried out to enhance the performance of the sea delay network comprehensively.

\section{ACKNOWLEDGMENT}

This paper is supported by the National Natural Science Foundation of China (61661018); the Key R \& D Program of Hainan Province (ZDYF2016010); Hainan Natural Science Fund Project (20166210); the Key R \& D Project of Higher Education of Hainan Provincial Department of Education(Hnky2016ZD-5); Hainan University Research Foundation Fund Project (kyqd1536); Hainan Province graduate students innovative research project (Hys2016-59). Hui $\mathrm{Li}$ is the corresponding author.

\section{REFERENCES}

[1] W. G. Chen, J. S. Yang, J. G. Ma, and S Li, "New developments in maritime communications: a comprehensive survey," China Communications, Vol. 9, Oct. 2012, pp. 31-42.

[2] Y. Bai, and W. C. Du, "A Mobile Communication System for marine vessels," China, CN103037542A, April 2013.

[3] A. J. Pullin, S. Presland,and C. Pattinson, "Using ship movement in the Irish sea for MANET evaluation," Second UKSIM European Symposium on Computer Modeling and Simulation, IEEE Press, Sept. 2008, pp. 394-399, doi: 10.1109/EMS.2008.50.

[4] S. S. Yang, Research of routing protocols in maritime mobile Ad hoc networks. Dalian Maritime University. 2011

[5] P. Nicholas, J. Pepper, C. Weaver, D. Gibbons, and M. Muratore. "Simulation and analysis of mobile Ad hoc network technology in the US marine corps infantry battalion," Military Operations Research. Vol. 18, 2013, pp. 19-35.

[6] S. Fowjiya, A. Udhayachandrika, and D. A. Kathirvel, "Architectural overview of delay tolerant network," International Journal of Engineering Sciences \& Research Technology, Vol. 2, Oct. 2013, pp. 2922-2926.

[7] K. Fall and S. Farrell, "DTN: an architectural retrospective," IEEE Journal on Selected Areas in Communications, Vol. 26, June 2008, pp. 828-836, doi:10.1109/JSAC.2008.080609.

[8] Q. Shao, Y. S. Ding, and Z. H. Hu, "Reliability Research on Channel Failure in DTN," Computer Simulation, Vol. 30, 2013, pp. 187-190.

[9] R. S. Mangrulkar, and M. Atique, "Routing protocol for delay tolerant network: A survey and comparison." IEEE International Conference on Communication Control and Computing Technologies, IEEE Press, Dec. 2010, pp. 210-215, doi: 10.1109/ICCCCT.2010.5670553.

[10] M. Thomas, S. Phand, and A. Gupta, "Using group structures for efficient routing in delay tolerant networks", Ad Hoc Networks, Vol. 7, 2009, pp. 344-362, doi: 10.1016/j.adhoc.2008.04.001.

[11] S. Zhou, L. Ying, and S. Tirthapura, "Delay, cost and infrastructure tradeoff of epidemic routing in mobile sensor networks," International Wireless Communications and Mobile Computing Conference, Iwcmc 2010, Caen, France, June 28 - July 2, 2010, pp. 1242-1246.

[12] S. Eshghi, M. H. R. Khouzani, S. Sarkar, N. B. Shroff, and S. S. Venkatesh, "Optimal energy-aware epidemic routing in DTNs," IEEE Transactions on Automatic Control, Vol. 60, June 2014, pp. 15541569, doi: 10.1109/TAC.2015.2396641.

[13] Y. H. Wu, S. Deng, and H. B. Huang, "Performance analysis of hoplimited epidemic routing in DTN with limited forwarding times," International Journal of Communication Systems, Vol. 28, August 2014, pp. 2035-2050, doi: 10.1002/dac.2845.

[14] T. Spyropoulos, K. Psounis, and C. S. Raghavendra, "Spray and wait: An efficient routing scheme for intermittently connected mobile networks," SIGCOMM'05 Workshops, August 22-26, 2005, Philadelphia, PA, USA, pp. 252-259, August 2005, doi: 10.1145/1080139.1080143.

[15] R. Geng, M. D. Tang, and X. H. Jiang, "Spray and Wait Routing Based on Relay-Probability in DTN," Journal of Northeastern University, Vol. 33, 2012, pp. 1698-1701. 
[16] H. Li, "Mobile connection routing under modified random waypoint models in delay tolerant mobile networks," Journal of Jilin University, Vol. 41, July 2011, pp. 297-303, doi: 10.13229/j.cnki.jdxbgxb2011.s1.029.

[17] H. O. Walther, "On Poisson's state-dependent delay," Discrete and Continuous Dynamical Systems, Vol. 33, January 2013, pp. 365-379, doi:10.3934/dcds.2013.33.365

[18] J. Zhao, P. Guo, H. Z. Deng, J. Wu, Y. J. Tan, and J. P. Li, "Modeling and analysis of performance reliability for communication networks based on traffic dynamics," Journal on Communications, Vol. 32, Aguest 2011, pp. 159-164.

[19] L. L. Jiang, G. H. Yang, Y. F. Dan, and H. Y. Wang, "Analysis of stability for networked control systems with large delays and packet losses," Application Research of Computers, Vol. 27, June 2010, pp. 2283-2286.

[20] L. J. Tang, Y. Li, Y. Chai, and X. Wang, "Buffer schedule algorithm combined with epidemic routing," Journal of Xidian University, Vol. 39, 2012, pp. 141-145. 